\title{
BMJ Open Efficacy, immunogenicity and safety of a recombinant tetravalent dengue vaccine (CYD-TDV) in children aged 2-17 years: systematic review and meta-analysis
}

\author{
Bruno Rodrigues Rosa, ${ }^{\circledR}$ Antonio José Ledo Alves da Cunha, \\ Roberto de Andrade Medronho
}

To cite: Rosa BR, Cunha AJLA, Medronho RA. Efficacy, immunogenicity and safety of a recombinant tetravalent dengue vaccine (CYD-TDV) in children aged 2-17 years: systematic review and meta-analysis. BMJ Open 2019;9:e019368. doi:10.1136/ bmjopen-2017-019368

- Prepublication history and additional material for this paper are available online. To view these files, please visit the journal online (http://dx.doi org/10.1136/bmjopen-2017019368).

Received 16 March 2018 Revised 19 December 2018 Accepted 20 December 2018

Check for updates

(C) Author(s) (or their employer(s)) 2019. Re-use permitted under CC BY-NC. No commercial re-use. See rights and permissions. Published by BMJ.

Instituto de Estudos em Saúde Coletiva, Universidade Federal do Rio de Janeiro, Rio de Janeiro, Brazil

Correspondence to Dr Bruno Rodrigues Rosa; eubrunorosa@gmail.com

\section{ABSTRACT}

Background Randomised controlled trials have evaluated the recombinant tetravalent dengue vaccine (CYD-TDV). However, individual results may have little power to identify differences among the populations studied. Objective To evaluate efficacy, immunogenicity and safety of CYD-TDV in the prevention of dengue in children aged 2-17 years.

Design Systematic review and meta-analysis.

Data sources MEDLINE (from 1950 to 5 December 2018), EMBASE (from 1947 to 5 December 2018) and Cochrane (from 1993 to 5 December 2018).

Eligibility criteria of studies Randomised trials comparing efficacy, immunogenicity and safety of CYDTDV with placebo or other vaccines for preventing dengue cases in children aged 2-17 years.

Outcome measures Efficacy, immunogenicity and safety of CYD-TDV.

Study appraisal and methods Calculations were made of relative risk (RR) and mean difference (MD) for dichotomous and continuous outcomes, respectively. All estimates were calculated considering a $95 \% \mathrm{Cl}$ estimate. A $p<0.05$ was considered statistically significant. Results Nine studies involving 34248 participants were included. The overall efficacy of CYD-TDV was $60 \%$ (RR 0.40 (0.30 to 0.54$)$ ). Serotype-specific efficacy of the vaccine was $51 \%$ for dengue virus type- 1 (DENV1) (RR 0.49 (0.39 to 0.63$)$ ); $34 \%$ for DENV-2 (RR 0.66 (0.50 to 0.86$)$ ); $75 \%$ for DENV-3 (RR 0.25 (0.18 to 0.35 )) and $77 \%$ for DENV-4 (RR 0.23 (0.15 to 0.34 )). Overall immunogenicity (MD) of CYD-TDV was 225.13 (190.34 to 259.93). Serotype-specific immunogenicity was: DENV-1: 176.59 (123.36 to 229.83); DENV-2: 294.21 (181.98 to 406.45); DENV-3: 258.78 (146.72 to 370.84) and DENV-4: 189.35 (141.11 to 237.59). The most common adverse events were headache and pain at the injection site. Limitations The main limitation of this study was unclear or incomplete data.

Conclusions and implications of key findings CYD-TDV is considered safe and able to partially protect children and adolescents against four serotypes of DENV for a 1-year period. Despite this, research should prioritise improvements in vaccine efficacy, thus proving higher long-term protection against all virus serotypes. PROSPERO registration number CRD42016043628.
Strengths and limitations of this study

- Systematic review with meta-analysis constitutes the highest level of evidence in health-making decisions.

- This systematic review evaluated the effects of recombinant tetravalent dengue vaccine in the prevention of dengue cases exclusively in children aged 2-17.

- Overall and dengue virus serotype-specific efficacy and immunogenicity of the vaccine were assessed.

- The lack of data compromised some more specific analyses (eg, subgroup analyses of efficacy of vaccine on different age groups).

\section{INTRODUCTION}

According to WHO, an estimated 50-100million dengue infections occur worldwide per year. ${ }^{2}$ This global figure has been updated by Bhatt et $a l_{,}^{2}$ who estimated there to be approximately 390 million (95\% CI 284 to 528) dengue infections per year, of which 96 million manifests clinically. The four serotypes of dengue virus type-1 (DENV-1 to DENV-4) are transmitted among humans by female mosquitoes mainly of the Aedes aegypti species. ${ }^{34}$ Recovery from infection by one dengue serotype provides lifelong immunity against that particular serotype. ${ }^{5}$ Severe dengue is a leading cause of death among children in Southeast Asian and Latin American countries. ${ }^{46}$

Differently, from what occurs with adults, the onset of dengue infection might go unnoticed in children, and severe infection may only be identified as the first clinical manifestation. Dengue-infected children usually worsen suddenly, hindering the identification of warning signs. ${ }^{7}$ Immune response in secondary dengue infection is associated with severe disease due to a mechanism called antibody-dependent enhancement. ${ }^{89}$ Vector 
control measures and personal protection have failed to prevent DENV transmission in endemic countries. ${ }^{10}$ The development of vaccines holds substantial potential in controlling the disease, particularly in protecting children from infection. The vaccine should, therefore, induce long-lasting immunity and protect against all four DENV serotypes simultaneously. ${ }^{4-12}$

Despite the challenges in developing recombinant tetravalent dengue vaccines (CYD-TDVs), several potential vaccines are in advanced stages of development. ${ }^{43}$ The most advanced is Dengvaxia (CYD-TDV), also known as ChimeriVax, a chimeric tetravalent vaccine developed by Sanofi Pasteur that comprises recombinant, live, attenuated strains of the four serotypes of the DENV. ${ }^{11}{ }^{13-17}$ In this vaccine, the premembrane (prM) and envelope (E) protein genes of each of the four DENVs were inserted individually to replace those of the yellow fever virus in the yellow fever 17D vaccine backbone (YFV 17D). ${ }^{10} 1113$ 15-18 The strains used in CYD-TDV are genetically and phenotypically stable, non-hepatotropic and less neurovirulent than the strains used in YFV 17D. ${ }^{17}$ Results of preclinical studies suggest that live attenuated vaccines such as CYD-TDV have some advantages over other prospective dengue vaccines: the live attenuated vaccines act as agents of RNA replication, inducing humoral and cellular immune responses; a single-dose vaccination regimen may induce immune responses; and vaccines can be produced at a relatively low cost. ${ }^{19}$ In addition, CYD-TDV has been shown to induce controlled stimulation of human dendritic cells and other immune responses. ${ }^{17}$

There are two reasons why the effects of CYD-TDV were evaluated exclusively in individuals under 18 years of age: (1) dengue infection may have different clinical manifestations in children, which may affect the assessment of vaccine efficacy and (2) a change has been observed in the epidemiological pattern of dengue in Brazil in recent years, characterised by the occurrence of severe cases and a proportional increase in cases among children. Hence, there is an urgent need for an effective and safe vaccine particularly for the protection of children.

Although CYD-TDV has now been licensed in several countries, including Brazil and Mexico, ${ }^{20}$ there has been no systematic review to evaluate the efficacy, immunogenicity and safety of CYD-TDV exclusively in the under 18 population. This indicates a clear need for a comprehensive analysis of efficacy, immunogenicity and safety of CYD-TDV in the prevention of dengue infection in children. Therefore, we conducted a systematic review of randomised controlled trials (RCTs) to evaluate efficacy, immunogenicity and safety of CYD-TDV versus placebo or any other vaccine in the prevention of dengue infection in children aged $2-17$.

\section{METHODS AND DESIGN}

\section{Eligibility criteria}

To be eligible, the RCTs were also required to have compared CYD-TDV used in a regimen of three doses administered at 6-month intervals ${ }^{19}$ (intervention group) versus placebo or any other vaccine (control group). RCTs with no age restriction, that is, which also included adults were eligible for inclusion in this review assuming that separate analyses were performed for under 18 age groups, in which case-only data of the under 18 years were used in the analysis.

Potentially relevant RCTs were excluded from this review whenever they met any of the following exclusion criteria: (1) use of dengue vaccines whose formulation is different from that described above; (2) use of CYD-TDV in less than three doses; (3) inclusion of patients with immunodeficiency, chronic diseases and/or receiving any treatment that could interfere with the immune response to the vaccine and (4) clinical trials conducted exclusively in patients aged 18 years and above or that included children and adults but did not perform separate analyses for participants younger than 18 years.

The following outcomes were assessed: overall and serotype-specific CYD-TDV vaccine efficacy, immunogenicity and occurrence of adverse events (AEs). The immunogenicity of the vaccine was estimated by measuring the geometric mean titres (GMTs) for each DENV serotype at 28 days after administration of the third vaccine dose and subsequently comparing GMTs between the groups (intervention and control). GMTs were obtained using the $50 \%$ plaque reduction neutralisation test.

The occurrence of $\mathrm{AE}$ was recorded as rate of local and/or systemic AE, rate of serious AEs (SAEs) and rate of vaccine-related $\mathrm{AE}$.

\section{Information sources}

A search was carried out by two independent reviewers (BRR and RAM) using MEDLINE (accessed via PubMedfrom 1950 to 5 December 2018), EMBASE (from 1947 to 5 December 2018) and Cochrane electronic databases (from 1993 to 5 December 2018). Handsearching was also carried out in reference lists of all selected RCTs to identify additional potentially relevant studies. Reports of ongoing studies were checked on the ClinicalTrials. gov database (https://clinicaltrials.gov). No limits were applied for the search period.

\section{Search}

Full electronic search strategy was used on MEDLINE and Cochrane electronic databases: "dengue" [MeSH Terms] OR (breakbone fever) OR (fever, breakbone) OR (classical dengue fever) OR (classical dengue fevers) OR (dengue fever, classical) OR (dengue fever) OR (fever, dengue) OR (break-bone fever) OR (break bone fever) OR (fever, break-bone) OR (classical dengue) OR (classical dengues) OR (dengue, classical) AND "vaccines" [MeSH Terms] OR (attenuated vaccines) OR (vaccines, live, attenuated) OR (chimeriVax-dengue 4 vaccine).

\section{Study selection}

Recommendations in the Cochrane Handbook for Systematic Reviews of Interventions were followed-V.5.0.2. Two 
reviewers (BRR, RAM) independently screened search results for potentially relevant trials to be included in the review and full-text articles of these trials were retrieved. The inclusion criteria were independently employed through the use of an eligibility form. Individual differences were resolved by either consensus decision-making or through the input of a third member (AJLAC) of the review team. Data were not included in the review before the full decision-making process was appropriately concluded. In addition, all excluded studies were listed and the reasons for their exclusion were duly justified. No limits were applied for either language or year of publication.

\section{Data collection process}

Potentially relevant articles were evaluated independently by two reviewers (BRR and RAM). Data from these papers were extracted through the use of a standardised form. A flow chart describing the study's selection process was created based on the Preferred Reporting Items for Systematic Reviews and Meta-analyses (see online supplementary table 1) model. ${ }^{21}{ }^{22}$ Whenever relevant data were found to be missing in the selected RCTs, the authors of these articles were sent an email requesting this information. Disagreements between reviewers were resolved by either consensus decision-making or with the input of a third team member.

\section{Data items}

Intervention was defined as administration of the CYD-TDV whose tetravalent formulation is characterised by the replacement of the YFV genome (used as backbone) with structural genes ( $\mathrm{prM}$ and $\mathrm{E}$ ) of each of the four DENV serotypes.

Efficacy was defined as the protective effect of CYD-TDV against symptomatic, virologically confirmed dengue achieved at 28 days after administration of the third dose and lasting for the subsequent 13 months, regardless of disease severity or virus serotype. ${ }^{23}{ }^{24}$ Studies based on less than a 13-month follow-up period were not included in the efficacy analysis.

Immunogenicity was defined as the ability of the vaccine to stimulate immune responses, such as antibody-mediated and cell-mediated immunity as well as immunological memory. ${ }^{23}$

Local AEs were defined as those occurring within 7 days of administration of any of the three vaccine doses and included, among others, pain, erythema and oedema at the injection site. Systemic AEs were defined as those persisting for 28 days or longer after administration of any of the three vaccine doses and included, among others, fever, headache, malaise, muscle pain and loss of appetite. The rate of SAE was estimated for all interventions used on the control group (including placebo).

\section{Assessment of risk of bias in selected individual studies}

The risk of bias in the studies selected was assessed independently by two reviewers (BRR and RAM), considering the following domains associated with risk of bias: (1) generation of an allocation sequence; (2) concealment of the allocation sequence; (3) blinding of participants, investigators and outcome assessors and (4) selective reporting of outcomes. Each of these items was classified into 'yes', 'no' or 'unclear' categories, indicating low, high or unknown risk of bias, respectively. The criteria used in this assessment were extracted from Cochrane Collaboration's tool for assessing the risk of bias entitled 'The Grading of Recommendations, Assessment, Development and Evaluation'. ${ }^{25} 26$

\section{Summary measures}

Dichotomous outcomes (efficacy and safety) were assessed using relative risk (RR). A $2 \times 2$ table was used to calculate RR. The Mantel-Haenszel (M-H) random-effects model estimated the pooled RR and associated 95\% CI. The M-H method provides a pooled RR across the strata of fourfold data. Continuous outcomes (immunogenicity) were assessed using mean difference (MD) analyses (a difference between two means). A random-effects model with the inverse variance method was used to estimate the pooled MD and associated 95\% CI. Both overall and serotype-specific CYD-TDV vaccine efficacies were calculated using the expression: (1-RR)x100. Results were compared using the $\chi^{2}$ test and z-test. All estimates were calculated considering a $95 \%$ CI. A $\mathrm{p}<0.05$ was considered statistically significant. ${ }^{27}$

\section{Synthesis of results}

A meta-analysis was performed when the same outcome was assessed in at least two RCTs. Meta-analysis is used to combine more than one trial and produce an average result. The purpose is to provide a more precise estimate of the effects of an intervention and reduce uncertainty. The $\chi^{2}$ test assesses whether observed differences in results between intervention group versus control group were compatible by chance alone. The z-test refers to the interventions summary effect in a meta-analysis where 0 indicates there is no effect (or no effect on average in a random-effects meta-analysis). Heterogeneity between studies was assessed using the $\mathrm{I}^{2}$ statistic, where $\mathrm{I}^{2}$ values $<25 \%$ indicated a low level of inconsistency, values of $25 \%-50 \%$ a moderate level of inconsistency and values $>50 \%$ a high level of inconsistency. ${ }^{27}$ All analyses were performed using the Review Manager software, V.5.3 (Cochrane). ${ }^{27}$

\section{Additional analyses}

Subgroup analyses were carried out for both efficacy and immunogenicity outcomes. An RR was calculated for each serotype-specific CYD-TDV vaccine efficacy. On the other hand, an MD was calculated for each serotype-specific CYD-TDV vaccine immunogenicity.

\section{Patient and public involvement}

Patients were not directly consulted during the planning of this research. However, following completion of the work, the findings shall be disseminated through 


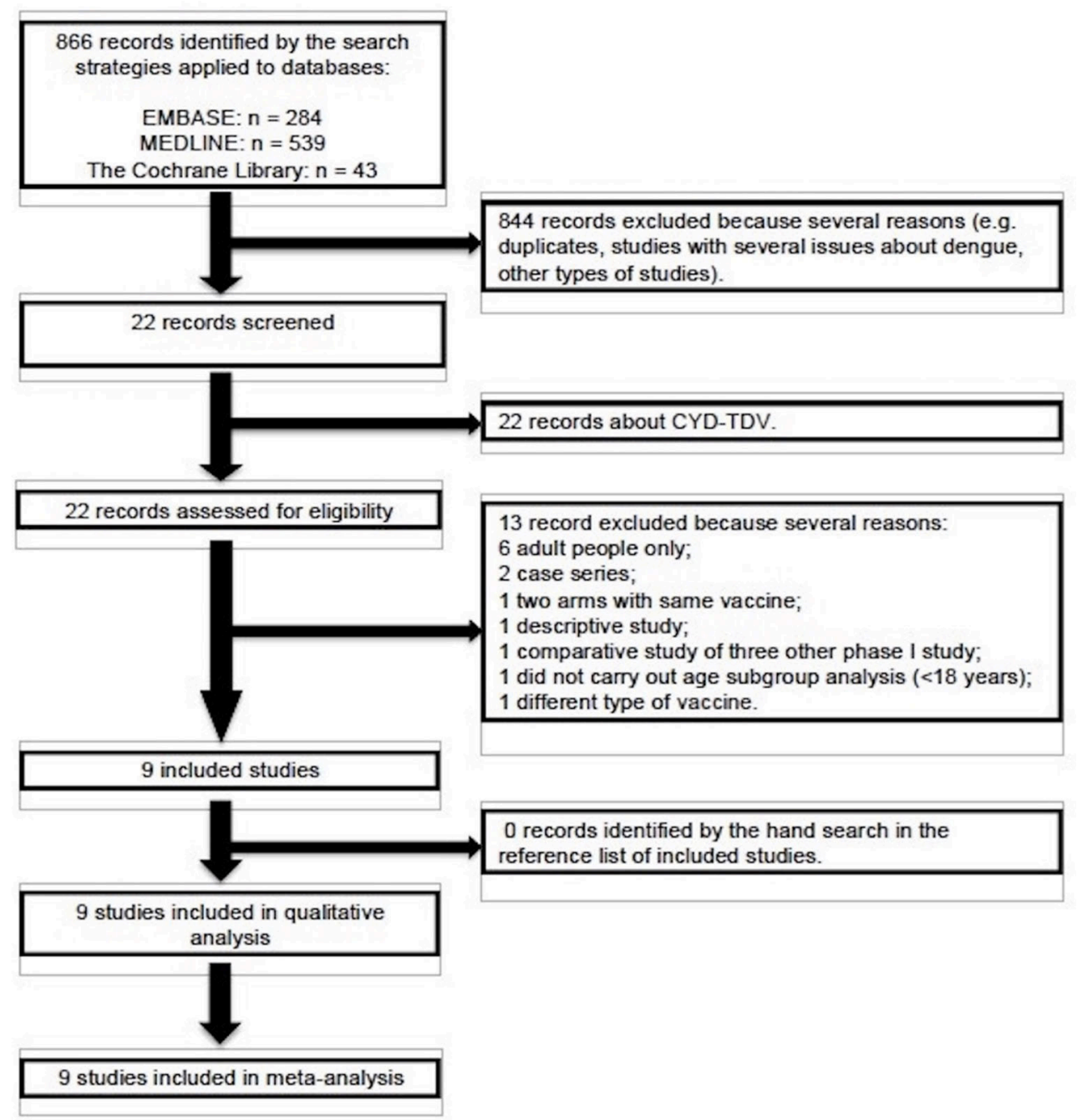

Figure 1 Study flow chart. CYD-TDV, recombinant tetravalent dengue vaccine.

open-access publications. Lay summaries shall also be made available on our institution's website.

\section{RESULTS}

\section{Study selection}

A total of nine RCTs ultimately met the selection criteria and were included in the systematic review involving 34248 participants (22916 in the intervention group vs 11332 in the control group). ${ }^{28-36}$ Figure 1 provides a flow chart of the study's identification and selection process as well as the reasons for exclusions. Additional studies were not obtained through the cross-checking of reference listings or the ClinicalTrials.gov database (no ongoing studies were found on the latter-all were RCTs that had already been published) table 1 shows the characteristics of studies that were included.

\section{Study characteristics}

The mean age of participants was 9.7, and the male to female ratio was similar overall (17977 females, 51.4\%). Two RCTs included adults but performed separate analyses for the under 18 group. ${ }^{30} 32$ All RCTs were conducted either in Southeast Asian countries (Indonesia, Malaysia, Philippines, Thailand, Vietnam and Singapore) or in Latin American countries (Colombia, Honduras, Mexico, Puerto Rico, Peru and Brazil), which are all dengue-endemic areas.

Most RCTs evaluated the immunogenicity and safety of CYD-TDV, while only three trials assessed the efficacy of the vaccine with a follow-up of 13 months after administration of the third dose. ${ }^{31} 3536$ Four trials compared CYD-TDV versus placebo (sodium chloride 0.9\%). ${ }^{2829} 3536$ In all other trials, the following standardised vaccines were administered to the control group: tetanus, diphtheria and acellular pertussis vaccines ${ }^{32}$; hepatitis $\mathrm{A}$ and influenza vaccines ${ }^{30}$; typhoid Vi polysaccharide and pneumococcal polysaccharide vaccines ${ }^{32}$; ; and rabies vaccine. ${ }^{31}$

\section{Risk of bias}

Overall, the methodological quality of the studies included in this review was considered good. Randomisation methods were appropriately defined in all RCTs, and there was no evidence of selective reporting of outcomes. However, information on blinding was not provided 


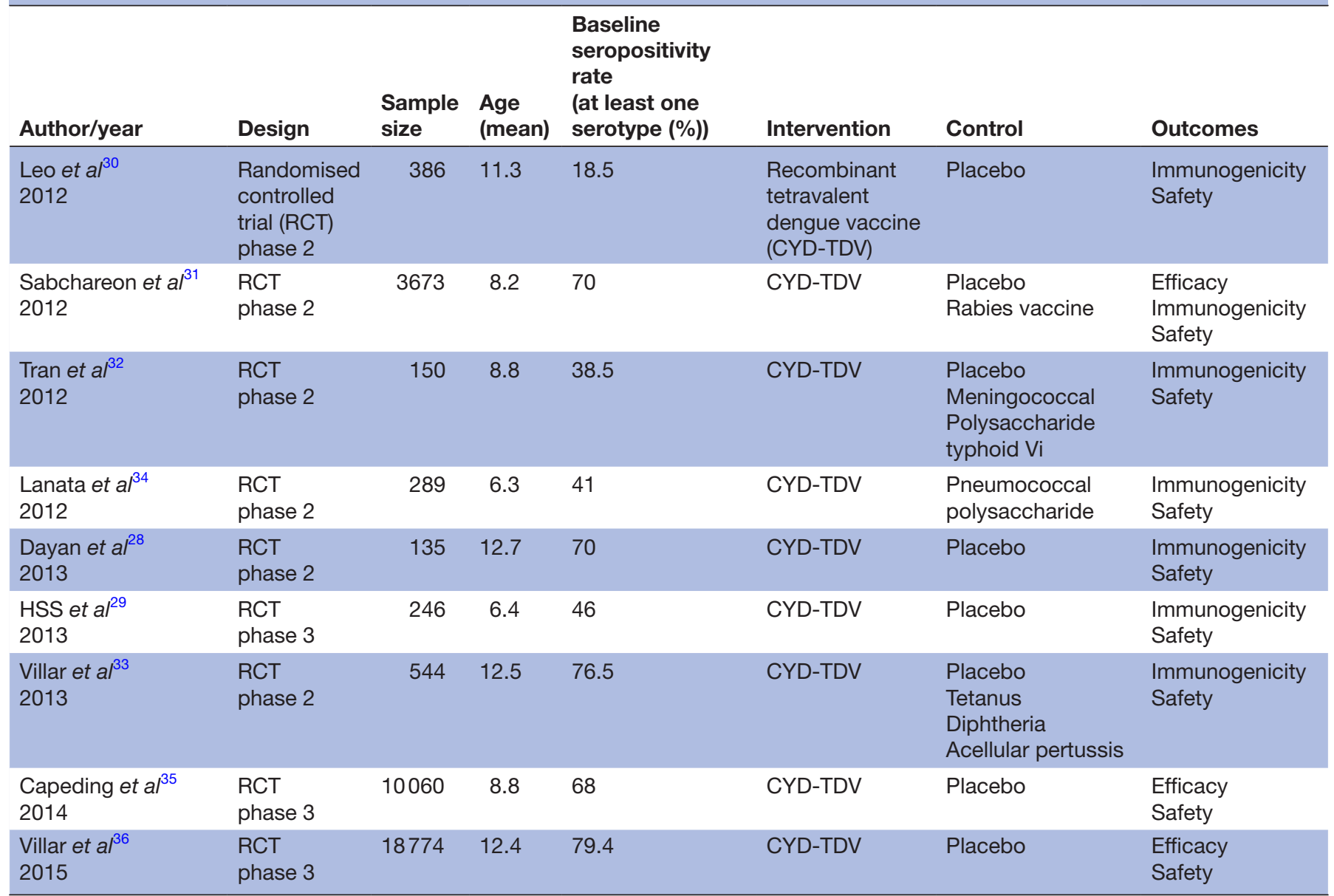

in four trials 28293234 and participant allocation was not concealed in two trials. ${ }^{29} 30$

\section{Results of individual studies}

Efficacy

Three RCTs assessed the efficacy of CYD-TDV, for a total of 31128 participants analysed (20841 in the CYD-TDV group vs 10287 in the control group). ${ }^{313536}$ The overall estimate of the effect of CYD-TDV was 0.40 (RR $0.40(95 \%$ CI 0.30 to 0.54$)$ ). CYD-TDV showed an efficacy of $60 \%$. Other three trials $^{32-34}$ reported dengue cases after the third CYD-TDV dose, however, it is unclear whether or not the authors assessed vaccine efficacy, that is, the text is vague about whether all participants were systematically evaluated for confirmation of the disease. The authors were contacted for clarification, yet replies were not received. These three RCTs were, therefore, not included in the meta-analysis. Serotype-specific efficacy showed that the CYD-TDV vaccine was more effective against DENV-4 (77\%-RR 0.23 (95\% CI 0.15 to 0.34$), \mathrm{p}<0.00001)$. On the other hand, CYD-TDV was less effective against DENV-2 (34\%-RR 0.66 (95\% CI 0.50 to 0.86$), \mathrm{p}<0.002)$. Figure 2 shows both overall and serotype-specific efficacy of CYD-TDV.

\section{Immunogenicity}

Six trials assessed immunogenicity as an outcome measure and these were included in the meta-analysis. ${ }^{28} 2931333436$ The results of the immunogenicity meta-analyses are shown in figure 3. The CYD-TDV overall immunogenicity was: $\mathrm{MD}=225.13$ (95\% CI 190.34 to 259.93). For all four DENV serotypes, the GMTs at day 28 after the third dose were higher in the CYD-TDV group: $\mathrm{MD}=176.59$ (95\% CI 123.36 to 229.83) for DENV-1; MD=294.21 (95\% CI 181.98 to 406.45$)$ for DENV-2; $\mathrm{MD}=258.78(95 \%$ CI 146.72 to 370.84) for DENV-3 and $\mathrm{MD}=189.35$ (95\% CI 141.11 to 237.59) for DENV-4. Overall, the heterogeneity among studies was considered moderate $(36.1 \%)$. However, serotype-specific immunogenicity analyses demonstrated a high level of inconsistency (range from 83\% (DENV-1) to 96\% (DENV-2 and DENV-3)).

\section{Safety (AE)}

Seven trials assessing safety as an outcome measure reported on local and systemic AE. ${ }^{28-3032-3436}$ The overall proportion of $\mathrm{SAE}$ was $5.2 \%$ in the CYD-TDV group (1283/24768 participants) and 6.4\% in the control group (791/12250 participants) $(\mathrm{p}=0.006)$. Eight children had vaccine-related $\mathrm{AE}$ (five in the intervention group and three in the control group). The AEs reported in the 


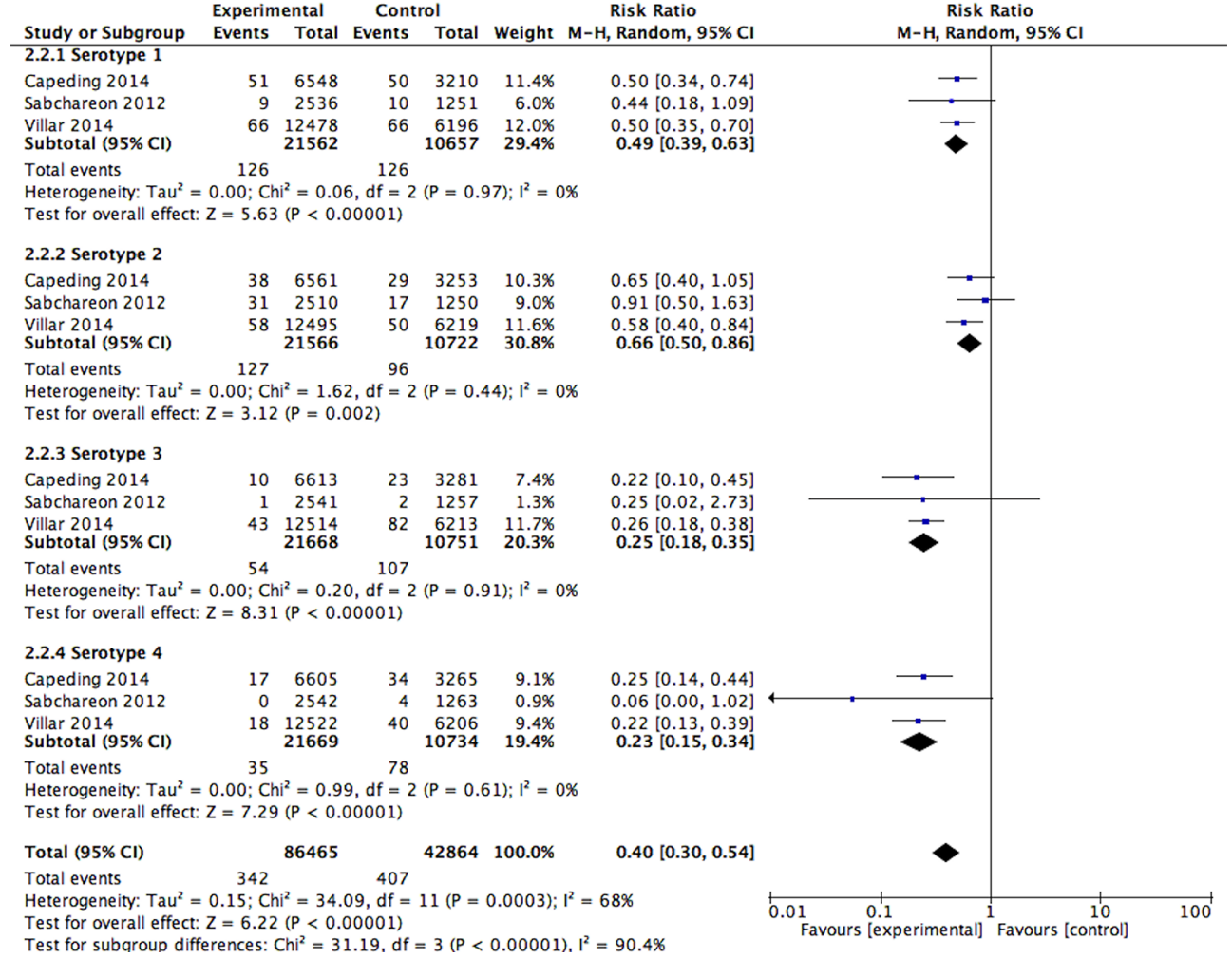

Figure 2 Overall and serotype-specific DENV efficacy analysis. DENV, dengue virus; M-H, Mantel-Haenszel.

\begin{tabular}{|c|c|c|c|c|c|c|c|c|c|}
\hline \multirow{3}{*}{$\begin{array}{l}\text { Study or Subgroup } \\
1.1 .1 \text { DENV-1 } \\
\text { Dayan } 2013\end{array}$} & \multicolumn{3}{|c|}{ Experimental } & \multicolumn{3}{|c|}{ Control } & \multirow[b]{2}{*}{ Weight } & \multirow{2}{*}{$\begin{array}{l}\text { Mean Difference } \\
\text { IV, Random, } 95 \% \mathrm{Cl}\end{array}$} & \multirow{2}{*}{$\begin{array}{l}\text { Mean Difference } \\
\text { IV, Random, } 95 \% \mathrm{Cl}\end{array}$} \\
\hline & Mean & & Total & Mean & & Total & & & \\
\hline & 267 & 513.3 & & 79.2 & & 49 & & $187.80[74.95,300.65]$ & 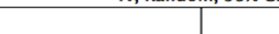 \\
\hline & 151 & 227.2 & 196 & 18.9 & 44.2 & 50 & $4.7 \%$ & $132.10[98.01,166.19]$ & - \\
\hline Lanata 2012 & 179 & 276.6 & 199 & 38.1 & 93.2 & 99 & & $140.90[98.31,183.49]$ & - \\
\hline Sabchareon 2012 & 146.1 & 401.7 & 197 & 23.9 & 64.8 & 99 & & $122.20[64.67,179.73]$ & - \\
\hline Villar 2013 & 320 & 668.6 & 401 & 106 & 251.3 & 199 & $4.0 \% 2$ & $214.00[139.83,288.17]$ & \\
\hline $\begin{array}{l}\text { Villar } 2014 \\
\text { Subtotal }(95 \% \mathrm{Cl})\end{array}$ & 395 & 767.4 & $\begin{array}{l}1301 \\
2393\end{array}$ & 121 & 269.6 & $\begin{array}{r}643 \\
1139\end{array}$ & $\begin{array}{l}4.5 \% \\
25.2 \%\end{array}$ & $\begin{array}{r}274.00[227.38,320.62] \\
176.59[123.36,229.83]\end{array}$ & \\
\hline \multicolumn{10}{|c|}{$\begin{array}{l}\text { Heterogeneity: } \text { Tau }^{2}=3448.89 ; \mathrm{Ch}^{2}=29.71, \mathrm{df}=5(\mathrm{P}<0.0001) ; \mathrm{I}^{2}=83 \% \\
\text { Test for overall effect: } Z=6.50(\mathrm{P}<0.00001)\end{array}$} \\
\hline \multicolumn{10}{|l|}{ 1.1.2 DENV- 2} \\
\hline Dayan 2013 & 544 & 973.6 & 99 & 132 & 325.5 & 49 & & $412.00[199.66,624.34]$ & \\
\hline HSS 2013 & 180 & 254.3 & 196 & 16.3 & 31 & 50 & $4.6 \%$ & $163.70[127.08,200.32]$ & - \\
\hline Lanata 2012 & 178 & 187.8 & 199 & 21.6 & 40.2 & 99 & $4.7 \%$ & 156.40[ & $=$ \\
\hline Sabchareon 2012 & & & 197 & & 180.5 & & & 257.80[ & \\
\hline Villar 2013 & 486 & 766.2 & 401 & 103 & 303.3 & 199 & $3.8 \% 3$ & $383.00[296$. & \\
\hline $\begin{array}{l}\text { Villar } 2014 \\
\text { Subtotal }(95 \% \mathrm{Cl})\end{array}$ & 574 & 837.2 & $\begin{array}{l}1301 \\
2393\end{array}$ & 129 & 263.5 & $\begin{array}{r}643 \\
1139\end{array}$ & $\begin{array}{r}4.48 \\
22.6 \%\end{array}$ & $445.00[395.16,494.84]$ & \\
\hline \multicolumn{10}{|c|}{$\begin{array}{l}\text { Heterogeneity: } \text { Tau }^{2}=17342.63 ; \mathrm{Ch}^{2}=125.62, \mathrm{df}=5(\mathrm{P}<0.00001) ; \mathrm{I}^{2}=96 \% \\
\text { Test for overall effect: } Z=5.14(\mathrm{P}<0.00001)\end{array}$} \\
\hline \multicolumn{10}{|l|}{ 1.1.3 DENV-3 } \\
\hline Dayan 2013 & 741 & 513.3 & 99 & 140 & 179 & 49 & $3.2 \% \mathrm{E}-\mathrm{l}$ & $601.00[488.15,713.85]$ & \\
\hline HSS 2013 & 151 & 227.2 & 196 & 18.9 & 44.2 & 50 & $4.7 \%$ & $132.10[98.01,166.19]$ & - \\
\hline Lanata 2012 & 179 & 276.6 & 199 & 38.1 & 93.2 & 99 & $4.5 \%$ & $140.90[98.31,183.49]$ & - \\
\hline Sabchareon 2012 & 146.1 & 401.7 & 197 & 23.9 & 64.8 & 99 & $4.3 \%$ & $122.20[64.67,179.73]$ & $\rightarrow$ \\
\hline Villar 2013 & 320 & 668.6 & 401 & 1062 & 251.3 & 199 & $4.0 \% 2$ & $214.00[139.83,288.17]$ & $\rightarrow$ \\
\hline $\begin{array}{l}\text { Villar } 2014 \\
\text { Subtotal } 95 \% \mathrm{Cl}\end{array}$ & 508 & 784.8 & 1301 & 1242 & 257.4 & 643 & $\begin{array}{r}4.5 \% \\
25.2 \%\end{array}$ & $384.00[336.94,431.06]$ & 4 \\
\hline \multicolumn{10}{|c|}{$\begin{array}{l}\text { Heterogeneity: } \operatorname{Tau}^{2}=18502.34 ; \mathrm{Ch}^{2}=137.03, \mathrm{df}=5(\mathrm{P}<0.00001) ; I^{2}=96 \% \\
\text { Test for overall effect: } Z=4.53(\mathrm{P}<0.00001)\end{array}$} \\
\hline \multicolumn{10}{|l|}{ 1.1.4 DENV-4 } \\
\hline Dayan 2013 & 432 & 532.6 & 99 & 33.4 & 51.5 & 49 & $3.4 \% 3$ & $398.60[292.70,504.50]$ & \\
\hline HSS 2013 & 114 & 125.4 & 196 & 10.9 & 15.1 & 50 & $4.8 \%$ & $103.10[85.05,121.15]$ & - \\
\hline Lanata 2012 & 184 & 181 & 199 & 16.7 & 25.5 & 99 & $4.7 \%$ & $167.30[141.66,192]$ & $*$ \\
\hline Sabchareon 2012 & 155 & 248.1 & 197 & 19.4 & 49.6 & 99 & $4.6 \%$ & $135.60[99.60,171.60]$ & $=$ \\
\hline Villar 2013 & 273 & 329.7 & 401 & 42.8 & 76.8 & 199 & $4.7 \% 2$ & $230.20[196.21,264.19]$ & - \\
\hline $\begin{array}{l}\text { Villar } 2014 \\
\text { Subtotal }(95 \% \mathrm{Cl})\end{array}$ & 241 & 279 & $\begin{array}{l}1301 \\
2393\end{array}$ & 44.3 & ${ }^{73.5}$ & $\begin{array}{r}643 \\
1139\end{array}$ & $\begin{array}{r}4.8 \% \\
27.0 \%\end{array}$ & $\begin{array}{l}196.70[180.51,212.89] \\
189.35[141.11,237.59]\end{array}$ & 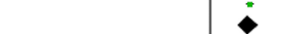 \\
\hline \multicolumn{10}{|c|}{$\begin{array}{l}\text { Heterogeneity: Tau }=3174.60 ; \mathrm{Chi}^{2}=95.29, \mathrm{df}=5(\mathrm{P}<0.00001) ; \mathrm{I}^{2}=95 \% \\
\text { Test for overall effect: } Z=7.69(\mathrm{P}<0.00001)\end{array}$} \\
\hline \multirow{2}{*}{\multicolumn{9}{|c|}{$\begin{array}{l}\text { Total }(95 \% \mathrm{Cl}) \\
\text { Heterogeneity: Tau }{ }^{2}=64771.30 ; \mathrm{Chi}^{2}=417.67 \text {, df }=23(\mathrm{P}<0.000001) ; \mathrm{I}^{2}=94 \% \\
\text { Test for overall effect: } \mathrm{Z}=12.68(\mathrm{P}<0.00001)\end{array}$}} & 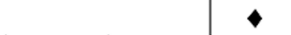 \\
\hline & & & & & & & & & $\begin{array}{l}000-500 \\
\text { Favours (cor }\end{array}$ \\
\hline
\end{tabular}

Figure 3 Overall and serotype-specific DENV immunogenicity analysis. CYD-TDV, recombinant tetravalent dengue vaccine; DENV, dengue virus; IV, inverse variance. 
Table 2 Safety analyses of the vaccine

\begin{tabular}{|c|c|c|c|c|c|c|c|c|}
\hline \multirow[b]{2}{*}{ Adverse events } & \multirow{2}{*}{$\begin{array}{l}\text { No of } \\
\text { studies }\end{array}$} & \multicolumn{2}{|l|}{ Intervention } & \multicolumn{2}{|l|}{ Control } & \multirow[b]{2}{*}{ RR (95\% Cl) } & \multirow{2}{*}{$\begin{array}{l}\text { Test for } \\
\text { heterogeneity, } \%\end{array}$} & \multirow[b]{2}{*}{$P$ value } \\
\hline & & $\mathrm{n} / \mathrm{N}$ & $\%$ & $\mathrm{n} / \mathrm{N}$ & $\%$ & & & \\
\hline \multicolumn{9}{|l|}{ Systemic reaction } \\
\hline Fever & 7 & $375 / 2683$ & 13.9 & $124 / 1224$ & 10.1 & 1.26 (1.03 to 1.53$)$ & $I^{2}=7$ & 0.03 \\
\hline Headache & 7 & $1015 / 2684$ & 37.8 & $408 / 1226$ & 33.2 & 1.10 (1.01 to 1.20$)$ & $I^{2}=0$ & 0.04 \\
\hline Malaise & 7 & $760 / 2684$ & 28.3 & $276 / 1226$ & 22.5 & 1.18 (1.05 to 1.33$)$ & $I^{2}=0$ & 0.005 \\
\hline \multicolumn{9}{|l|}{ Site reaction } \\
\hline Pain & 7 & $890 / 2684$ & 33.1 & $360 / 1226$ & 29.3 & $1.06(0.60$ to 1.86$)$ & $I^{2}=95$ & 0.85 \\
\hline Erythema & 7 & $198 / 2684$ & 7.3 & $71 / 1226$ & 5.7 & 0.95 (0.75 to 1.20$)$ & $1^{2}=0$ & 0.68 \\
\hline Oedema & 7 & $158 / 2684$ & 5.8 & $76 / 1226$ & 6.1 & 0.72 (0.47 to 1.10$)$ & $I^{2}=46$ & 0.13 \\
\hline $\begin{array}{l}\text { Serious adverse } \\
\text { events }\end{array}$ & 9 & $1283 / 24768$ & 5.2 & $791 / 12.250$ & 6.4 & 0.84 (0.75 to 0.95$)$ & $I^{2}=20$ & 0.006 \\
\hline
\end{tabular}

intervention group were asthma attacks, allergic urticaria, peripheral polyneuropathy, seizures ${ }^{36}$ and disseminated encephalomyelitis. ${ }^{35}$ The AEs reported in the control group were transient visual disturbances, ${ }^{36}$ palsy of the seventh cranial nerve ${ }^{29}$ and an unspecified adverse reaction to the rabies vaccine. ${ }^{31}$ Results of the safety analyses are shown in table 2.

All systemic AEs were significantly more frequent in the CYD-TDV group. Headache was the most common systemic $\mathrm{AE}$ in this group, accounting for $37.8 \%$ of cases versus $33.2 \%$ in the control group. For systemic $\mathrm{AE}, \mathrm{I}^{2}$ values were always below $25 \%$, indicating low statistical heterogeneity between studies. There was no significant difference in local AEs between groups. The most common local $\mathrm{AE}$ was pain at the injection site, accounting for $33.1 \%$ of cases in the CYD-TDV group versus $29.3 \%$ in the control group. All local AEs except oedema had $\mathrm{I}^{2}$ values $<50 \%$, indicating moderate statistical heterogeneity between studies.

\section{DISCUSSION}

\section{Summary of evidence}

CYD-TDV has proven to be relatively safe. Nine RCTs have demonstrated that CYD-TDV causes significantly less SAEs than other vaccines currently administered to children (such as tetanus and diphtheria). Also, only five participants had vaccine-related AEs. WHO Global Advisory Committee on Vaccine Safety (GACVS) has presented new evidence indicating that an increased risk of hospitalisation (and severe disease) by dengue affects vaccinated subjects who are naive to wild dengue infection prior to vaccination. This corroborates prior hypotheses suggesting that immune priming from natural or other stimulation such as immunisation with the dengue vaccine can lead to a higher risk of severe dengue disease on secondary exposure to wild DENVs. ${ }^{37}$

Despite there being a relatively small number of hospitalised dengue cases in the first year of study (Capeding et $a l^{35}$ ), GACVS highlighted the importance of understanding the potential factors associated with this increased RR of hospitalisation (and especially severe dengue). Among other hypotheses, confirmation of the serostatus at the time of vaccination is crucial: first, given the lower vaccine efficacy in participants who were serologically naive; second, considering the potential risk of immune enhancement among previously infected subjects. ${ }^{38}$

Immunogenicity was higher for DENV-2 and DENV-3. Even given these two serotypes, such findings were not perceived as enough to provide a true representation of the efficacy of CYD-TDV. This outcome was assessed in three RCTs included in this review. ${ }^{31} 3536$ Although four other studies assessed vaccine efficacy after the third dose, they followed the participants for only 6 months. ${ }^{28} 293233$ Had these studies been included in the present review, there might have been a reduction in the estimated time for vaccine effects. A significant heterogeneity shown in DENV serotype-specific analyses does not necessarily lead to the true intervention effect. However, it does that the studies did not all estimate the same quantity. A hypothesis that explains this finding may be the different populations of the studies. Differences between studies in terms of methodological factors (eg, use of blinding and concealment of allocation), clinical issues (eg, different populations) or even divergences in the way the outcomes are defined and measured, might expectedly lead to differences in the intervention effects observed. ${ }^{25}$

CYD-TDV had an efficacy of $60 \%$ against the four serotypes of DENV after the third vaccine dose and over a 13-month follow-up period. Despite representing an advance, this rate is considered low, given that the protective effect of the vaccine may either be compromised by the virus serotype or by the seronegative status prior to vaccination. Villar $e t a l^{36}$ found that CYD-TDV had greater efficacy in children who were seropositive at the time of recruitment than in those who were seronegative $\left(83.7 \%\right.$ vs $43.2 \%$, respectively).$^{36}$ Although there is no evidence that CYD-TDV can cause the disease, administration of CYD-TDV to a subgroup of dengue-naïve individuals may generate a group more 
susceptible to secondary episodes of severe dengue. As CYD-TDV does not result in a balanced immune response among the serotypes of the virus, the use of this vaccine on the subgroup of seronegative children may produce individuals who are susceptible to one or more serotypes whose immunogenicity of the vaccine is low. Should such individuals come into contact with the wild virus of these serotypes with low immunogenicity, cases of severe dengue may occur as a result of augmentation of the viral response.

Serotype-specific efficacy of vaccine ranged from $34 \%$ (DENV-2) to $77 \%$ (DENV-4). The efficacy of vaccine against DENV-1 and DENV-2 serotypes of DENV was low. In the two most extensive studies (Asian and Central/ South American trials), all four dengue serotypes contributed to the overall efficacy during the active phase and although immunogenicity was higher for DENV-2, this serotype was the one against which CYD-TDV had the lowest protective effect $(34 \%$ - RR $0.66(95 \%$ CI 0.50 to $0.86)$ ). The weak efficacy of the DENV-2 component of CYD-TDV might be explained by the phenomenon of viral diversity ${ }^{39}$ Viral diversity represents the geographical variants of same the virus, being characterised by the presence of structural or genetic variation. This heterogeneity may compromise the DENV optimal recognition by the antibody. ${ }^{40}$

Two systematic reviews evaluated the effects of CYD-TDV on the prevention of dengue infection. ${ }^{14}{ }^{39}$ da Costa et $\mathrm{al}^{14}$ conducted a meta-analysis to analyse the effects of CYD-TDV in adults and children in seven RCTs (two RCTs included participants aged 2-45 years). These authors evaluated vaccine efficacy based on five RCTs with a maximum follow-up of 6 months after administration of the third dose, although in four of those trials assessing these outcomes was not the aim. da Costa et $a l^{14}$ did not include two other recently published RCTs ${ }^{35} 36$ that performed efficacy analysis and included 27662 participants under 18 years of age. ${ }^{14}$ However, da Costa et $a l^{14}$ reported an efficacy of 59\% for CYD-TDV. Regarding SAEs, a significantly higher rate was found in the control group in both their study and ours.

In a similar study to our review, Malisheni et $a l^{39}$ included the same number of studies (nine) and estimated vaccine efficacy percentages close to those achieved here $(54 \%)$. Nevertheless, safety and immunogenicity outcomes were different. Malisheni $e t a l^{39}$ found the highest GMTs for DENV-3 (439.7, 95\% CI 331.7 to 547.7 ), while we have reported the highest GMT for DENV-2. In addition, in the safety analysis, the authors reported that CYD-TDV leads to significantly more local AEs than the control (RR 1.1,95\% CI 1.04 to $1.17 ; \mathrm{p}=0.001)$. One possible hypothesis to explain these differences is the number of studies considered by the authors. Seven RCTs evaluated the immunogenicity of CYD-TDV, but the authors considered only three for the analyses of DENV-2, DENV-3 and DENV-4. Likewise, local AE considered data from five studies (in the case of pain only three studies were considered), while only seven RCTs published sufficient data for safety analysis of CYD-TDV.

\section{Limitations}

The main limitation of this study was unclear or incomplete data, for instance, two RCTs that assessed immunogenicity could not be included in the meta-analysis. ${ }^{30} 32$ In both trials, the authors published only the combined GMT for the four serotypes of DENV, thus precluding statistical analysis based on the GMT for each virus serotype. In another study, ${ }^{35}$ only the rate of febrile episodes was reported for the two study groups. No clear response was received about this issue, and the author informed that no additional data would be provided.

Second, long-term analyses were unable to be performed given that the participants of the selected clinical efficacy trials were followed only for 1 year after the third vaccine dose. Long-term analyses should be in accordance with WHO recommendation to perform a thorough assessment of the effects of the vaccine in order to ensure that the immune response to vaccination does not predispose individuals to severe disease and that the risk of severe disease does not increase with time. These events may occur due to a decrease in the levels of vaccine-induced antibodies, as measured by their GMTs, in persons in whom immunity has not been naturally boosted. ${ }^{41}$

\section{CONCLUSIONS}

Dengue is a complex disease. Although progress has been made in recent years, it is necessary to conduct further research so as to achieve better vaccine efficacy. CYD-TDV is considered effective, safe and able to protect children against DENV-3 and 4 serotypes for 1 year. Nevertheless, further studies with long-term analyses are required. Research should give priority to improvements in vaccine efficacy, thus providing long-term protection against all four virus serotypes.

Acknowledgements We thank Fundação de Amparo à Pesquisa do Estado do Rio de Janeiro (FAPERJ) for having supported the English language translation of this manuscript.

Contributors BRR conceived and designed the study, conducted the literature search and acquired the data. RAM analysed and interpreted the data and conducted the literature search. AJLAC reviewed and prepared the first draft of the manuscript. All authors edited the manuscript and approved the final version for submission. This manuscript represents a valid work and has not yet been published nor is being considered for publication elsewhere.

Funding The authors have not declared a specific grant for this research from any funding agency in the public, commercial or not-for-profit sectors.

Competing interests None declared.

Patient consent for publication Not required.

Provenance and peer review Not commissioned; externally peer reviewed. Data sharing statement № additional data are available.

Open access This is an open access article distributed in accordance with the Creative Commons Attribution Non Commercial (CC BY-NC 4.0) license, which permits others to distribute, remix, adapt, build upon this work non-commercially, and license their derivative works on different terms, provided the original work is properly cited, appropriate credit is given, any changes made indicated, and the use is non-commercial. See: http://creativecommons.org/licenses/by-nc/4.0/. 


\section{REFERENCES}

1. World Health Organization. Dengue: guidelines for diagnosis, treatment, prevention and control. World Health Organization, New edition, 2009. WHO/HTM/NTD/DEN/2009.1. Available at: http:// www.who.int/tdr/publications/ documents/dengue-diagnosis.pdf/ (accessed 27 Sep 2015).

2. Bhatt S, Gething PW, Brady OJ, et al. The global distribution and burden of dengue. Nature 2013;496:504-7.

3. World Health Organization. Guidelines for plaque reduction neutralization testing of human antibodies to dengue viruses. World Health Organization, 2007. WHO/IVB/07.07. Available at: www.who. int/vaccines-documents/ (accessed 15 Jun 2014).

4. World Health Organization. Media Centre. Dengue and severe dengue. Fact sheet $\mathrm{N}^{\circ} 117$, January 2012. Available at: http://www. who.int/mediacentre factsheets/fs117/en/ (accessed 17 May 2014).

5. Gubler DJ. Dengue and dengue hemorrhagic fever. Clin Microbiol Rev 1998;11:480-96.

6. Kittigul L, Pitakarnjanakul P, Sujirarat D, et al. The differences of clinical manifestations and laboratory findings in children and adults with dengue virus infection. J Clin Virol 2007;39:76-81.

7. Ministério da Saúde. Secretaria de Vigilância em Saúde. Diretoria Técnica de Gestão. Dengue: diagnóstico e manejo clínico: adulto e criança/Ministério da Saúde, Secretaria de Vigilância em Saúde, Diretoria Técnica de Gestão. 4th edn. Brasília: Ministério da Saúde, 2013;80:il.

8. Halstead S, O'Rourke EJ. Dengue viruses and mononuclear phagocytes. I. Infection enhancement by non-neutralizing antibody. $J$ Exp Med 1977;146:201-17.

9. Schiøler Karin L, McCarty Craig W. Vaccines for preventing dengue infection (Protocol for a Cochrane Review). In: The Cochrane Library. $2014 ; 7$.

10. World Health Organization. Guidelines on the quality, safety and efficacy of dengue tetravalent vaccines (live, attenuated). World Health Organization, 2011. Available at: http://www.who. int/biologicals/vaccines/Dengue_DB_5_April_2012_clean.pdf/ (accessed 08 Mar 2014).

11. Durbin AP, Whitehead SS. Dengue vaccine candidates in development. Curr Top Microbiol Immunol 2010;338:129-43.

12. Whitehead SS, Blaney JE, Durbin AP, et al. Prospects for a dengue virus vaccine. Nat Rev Microbiol 2007;5:518-28.

13. Bentsi-Enchill AD, Schmitz J, Edelman R, et al. Long-term safety assessment of live attenuated tetravalent dengue vaccines: deliberations from a WHO technical consultation. Vaccine 2013;31:2603-9.

14. da Costa VG, Marques-Silva AC, Floriano VG, et al. Safety, immunogenicity and efficacy of a recombinant tetravalent dengue vaccine: A meta-analysis of randomized trials. Vaccine 2014;32:4885-92.

15. Sinha G. Sanofi's dengue vaccine first to complete phase 3. Nat Biotechnol 2014;32:605-6.

16. Harenberg A, Begue S, Mamessier A, et al. Persistence of Th1/ Tc1 responses one year after tetravalent dengue vaccination in adults and adolescents in Singapore. Hum Vaccin Immunother 2013;9:2317-25.

17. Guy B, Barrere B, Malinowski C, et al. From research to phase III: preclinical, industrial and clinical development of the Sanofi Pasteur tetravalent dengue vaccine. Vaccine 2011;29:7229-41.

18. Guy B, Guirakhoo F, Barban V, et al. Preclinical and clinical development of YFV 17D-based chimeric vaccines against dengue, West Nile and Japanese encephalitis viruses. Vaccine 2010;28:632-49.

19. Bhamarapravati N, Sutee $\mathrm{Y}$. Live attenuated tetravalent dengue vaccine. Vaccine 2000;18:44-7.

20. World Health Organization. Summary of the April 2016 meeting of the Strategic Advisory Group of Experts on immunization (SAGE). World Health Organization, 2016. Available at: http://www.who.int/ immunization/sage/meetings/2016/april/ SAGE_April_2016_Meeting_ Web_summary.pdf

21. Liberati A, Altman DG, Tetzlaff J, et al. The PRISMA statement for reporting systematic and meta-analyses of studies that evaluate interventions: explanation and elaboration. PLoS Med 2009;6:1-28.
22. Moher D, Liberati A, Tetzlaff J, Altman DG. Preferred reporting items for systematic reviews and meta-analyses: the PRISMA statement. Ann Intern Med 2009;151:264-9.

23. World Health Organization. Guidelines for the clinical evaluation of dengue vaccines in endemic areas. World Health Organization, 2008. WHO/IVB/08.12. Available at: http://apps.who.int/iris/bitstream/ 10665/69850/1/WHO_IVB_08.12_eng. pdf/ (accessed 27 Sep 2015).

24. Hombach J. Guidelines for clinical trials of dengue vaccine in endemic areas. J Clin Virol 2009;46(S2):S7-9.

25. Higgins JPT, Green S, (eds). Cochrane Handbook for Systematic Reviews of Interventions Version 5.1.0 [updated March 2011]. The Cochrane Collaboration, 2011. Available at: www.cochranehandbook.org

26. Higgins JPT, Altman DG, Gotzsche PC, et al. The Cochrane Collaboration's tool for assessing risk of bias in randomised trials. BMJ 2011;343:d5928.

27. Higgins JPT, Thompson SG. Quantifying heterogeneity in a metaanalysis. Stat Med 2002;21:1539-58.

28. Dayan GH, Garbes P, Noriega F, et al. Immunogenicity and safety of a recombinant tetravalent dengue vaccine in children and adolescents ages 9-16 years in Brazil. Am J Trop Med Hyg 2013;89:1058-65.

29. HSS A-S, Koh M-T, Tan KK, et al. Safety and immunogenicity of a tetravalent dengue vaccine in healthy children aged 2-11 years in Malaysia: a randomized, placebo-controlled, Phase III study. Vaccine 2013;31:5814-21.

30. Leo YS, Wilder-Smith A, Archuleta S, et al. Immunogenicity and safety of recombinant tetravalent dengue vaccine (CYD-TDV) in individuals aged $2-45$ y Phase II randomized controlled trial in Singapore. Hum Vaccin Immunother 2012;8:1-13.

31. Sabchareon A, Wallace D, Sirivichayakul C, et al. Protective efficacy of the recombinant, live-attenuated, CYD tetravalent dengue vaccine in Thai schoolchildren: a randomised, controlled phase $2 \mathrm{~b}$ trial. Lancet 2012;380:1559-67.

32. Tran $\mathrm{NH}$, Luong $\mathrm{CQ}$, Tqh $\mathrm{V}$, et al. Safety and immunogenicity of recombinant, live attenuated tetravalent dengue vaccine (CYDTDV) in healthy vietnamese adults and children. $J$ Vaccines Vaccin 2012;3:1-7.

33. Villar LÁ, Rivera-Medina DM, Arredondo-García JL, et al. Safety and immunogenicity of a recombinant tetravalent dengue vaccine in 9-16 year olds: a randomized, controlled, phase II trial in Latin America. Pediatr Infect Dis J 2013;32:1102-9.

34. Lanata CF, Andrade T, Gil Al, et al. Immunogenicity and safety of tetravalent dengue vaccine in 2-11 year-olds previously vaccinated against yellow fever: randomized, controlled, phase II study in Piura, Peru. Vaccine 2012;30:5935-41.

35. Capeding MR, Tran NH, Hadinegoro SRS, et al. Clinical efficacy and safety of a novel tetravalent dengue vaccine in healthy children in Asia: a phase 3, randomised, observer-masked, placebo-controlled trial. Lancet 2014;384:1358-65.

36. Villar L, Dayan GH, Arredondo-García JL, et al. Efficacy of a tetravalent dengue vaccine in children in Latin America. $N$ Engl J Med Overseas Ed 2015;372:113-23.

37. World Health Organization. Global Vaccine Safety. GACVS Statement on Dengvaxia $₫(C Y D-T D V)$. World Health Organization, 2017. Available at: https://www.who.int/vaccine safety/committee/ GACVS-StatementonDengvaxia-CYD-TDV/en/ (accessed 15 Jul 2018).

38. World Health Organization. Weekly epidemiological record. Addendum to report of the Global Advisory Committee on Vaccine Safety (GACVS), 10-11 June 2015 ${ }^{1}$. Safety of CYD-TDV dengue vaccine. World Health Organization, 2015;34:421-32.

39. Malisheni M, Khaiboullina SF, Rizvanov AA, et al. Clinical Efficacy, Safety, and Immunogenicity of a Live Attenuated Tetravalent Dengue Vaccine (CYD-TDV) in Children: A Systematic Review with Metaanalysis. Front Immunol 2017;8:863.

40. Dejnirattisai W, Supasa $P$, Wongwiwat W, et al. Dengue virus serocross-reactivity drives antibody-dependent enhancement of infection with zika virus. Nat Immunol 2016;23.

41. Hadinegoro SR, Arredondo-García JL, Capeding MR, et al. Efficacy and long-term safety of a dengue vaccine in regions of endemic disease. N Engl J Med Overseas Ed 2015;373:1195-206. 